\title{
Neuromuscular Ultrasound Versus Electrophysiological Studies in Assessment of Posterior Tibial Nerve Neuropathy in Rheumatoid Arthritis Patient Mostafa A. Al-Dahan ${ }^{1}$, Hegazy M. Al-Tamimy ${ }^{2}$, Ahmed M. Fahmy ${ }^{3}$, Mahmoud I. Risha ${ }^{4}$ \\ 1,2,3,4 Department of Physical medicine \& Rheumatology and Rehabilitation, Faculty of Medicine, Al-Azhar University, Cairo, Egypt \\ Corresponding author: Mahmoud I. Risha; Mobile: 01200021223; Email: dr.m_risha@yahoo.com
}

\begin{abstract}
Background: Rheumatoid arthritis is a systemic rheumatic disease characterized by symmetrical, often erosive and deforming polyarthritis. Extra-articular manifestations occur in 10-20\% of patients, especially those with high titers of rheumatoid factor. Neuromuscular ultrasound is useful for the work up of posterior tibial nerve neuropathy. Diagnosis of posterior tibial neuropathy is based on a combination of characteristic symptoms and electrophysiological abnormalities. Recently, neuromuscular ultrasound has become an attractive complement to electrodiagnostic (EDX) studies in the evaluation of peripheral nerves.
\end{abstract}

Aim of the Work: To evaluate neuromuscular ultrasound versus electrophysiological studies in assessment of posterior tibial nerve neuropathy in rheumatoid arthritis patients.

Patients and Methods: The subjects included in our study functionally were divided into two groups. Group (A): Thirty Egyptian patients with Rheumatoid Arthritis (RA), all fulfilled the American College of Rheumatology (ACR) 2010 criteria for diagnosis of RA and ACR criteria for classification of rheumatoid arthritis (1987). All were over the age of sixteen at time of diagnosis. They were recruited from Physical Medicine, Rheumatology and Rehabilitation Department at Al-Hussein and Sayed Galal University Hospitals during the period from November 2017 to April 2018. They were complaining of burning pain or paresthesia on the plantar aspect of the foot and toes. Group (B): Thirty healthy individuals, age and sex matched, were used as a control group after an informed consent from all subjects.

Results: Neuromuscular ultrasound had a complementary role in the diagnosis of posterior tibial nerve neuropathy. The combined use of electrophysiology with neuromuscular ultrasonography further confirmed the diagnosis of posterior tibial nerve neuropathy.

Conclusion: Neuromuscular ultrasound became an attractive complement to electrodiagnostic (EDX) studies in the evaluation of posterior tibial nerve neuropathy.

Keywords: Rheumatoid arthritis, neuromuscular ultrasound, nerve conduction studies.

\section{INTRODUCTION}

Rheumatoid arthritis is a systemic rheumatic disease characterized by symmetrical, often erosive and deforming polyarthritis. Extra-articular manifestations occur in $10-20 \%$ of patients, especially those with high titers of rheumatoid factor. Extra articular pathology includes bursitis, tendonitis, neuritis, and vasculitis ${ }^{(1)}$.

Vasculitic neuropathy in rheumatoid arthritis is a sequential process of inflammation, thrombosis and ischemic injury to vasa nervosum, especially involving epineural arteritis of nerve ${ }^{(2)}$.

Clinical presentations include entrapment neuropathy that is one of the commonest types of carpal tunnel syndrome, tarsal tunnel syndrome (TTS), ulnar neuropathy at the elbow (cubital tunnel syndrome), femoral neuropathy, peroneal neuropathy, combined sensorimotor polyneuropathy and mononeuritis multiplex ${ }^{(3)}$.

The symptoms of posterior tibial nerve neuropathy include pain and numbness in the sole of the foot, cramping pain, tingling, sensation of tightness, worsening of symptoms with prolonged standing and walking and signs of entrapment include hyperthesia, weakness, hypoesthesia and Tinel's sign ${ }^{(4)}$.
The posterior tibial nerve is a branch of the sciatic nerve with a nerve root supply of L4, L5, S1, S2 and $\mathrm{S} 3$. The nerve enters the leg between the two heads of the gastrocnemius muscle and the nerve lies deep to the soleus muscle in the posterior compartment of the leg. In the lower leg the nerve travels between the flexor digitorum longus and the flexor hallucis longus. It then travels behind the medial malleolus through the proximal tarsal tunnel where it divides into its terminal branches, the medial plantar nerve and the lateral plantar nerve ${ }^{(5)}$.

Evaluation mainstays of the rheumatoid foot include both electrophysiological studies and imaging techniques. Electrophysiological studies are used to assess the peripheral electrophysiological changes in the rheumatoid feet ${ }^{(6)}$.

Neuromuscular ultrasound is useful for the work up of posterior tibial nerve neuropathy. It can often locate the exact site of nerve entrapment and can provide useful information on the cause of nerve damage in the tunnel and address the pathology of tarsal tunnel syndrome, such as perineural tissue vascularity and concomitant tendon pathology ${ }^{(7)}$.

\section{AIM OF THE WORK}

The aim of the work was to evaluate neuromuscular ultrasound versus electrophysiological 
studies in assessment of posterior tibial nerve neuropathy in rheumatoid arthritis patients.

\section{PATIENTS AND METHODS}

The subjects included in our study were functionally divided into two groups. Group (A): Thirty Egyptian patients with Rheumatoid Arthritis (RA). All fulfilled the American College of Rheumatology (ACR) 2010 criteria for diagnosis of RA and ACR criteria for classification of rheumatoid arthritis (1987). All were over the age of sixteen at time of diagnosis. They were recruited from Physical Medicine, Rheumatology and Rehabilitation Department at Al-Hussein and Sayed Galal University Hospitals during the period from November 2017 to April 2018 They were complaining of burning pain or paresthesia on the plantar aspect of the foot and toes. Group (B): Thirty healthy individuals, age and sex matched, were used as a control group after an informed consent from all subjects. The study was approved by the Ethics Board of Al-Azhar University.

\section{Patient's selection}

Inclusion criteria: Over the age of sixteen at time of diagnosis. All rheumatoid patients were diagnosed according to American College of Rheumatology (ACR) 2010 criteria and ACR criteria for classification of rheumatoid arthritis (1987). All patients must be complaining of burning pain or paresthesia on the plantar aspect of the foot and toes.

Exclusion criteria: Foot trauma and fractures. Congenital or post-traumatic foot deformity. Varicose veins and deep venous thrombosis. Lower limb edema. Patients well known to have neurological disease with peripheral neuropathy as example (Guillain Barre Syndrome). Systemic diseases (other than RA) e.g., Diabetes mellitus, hereditary neuropathies.

\section{Investigations:}

(1) Clinical evaluation: included full history taking, clinical examination, musculoskeletal examination, neurological examination, disease activity by Disease activity score (DAS) 28 .

(2) Laboratory evaluation in the form of: CBC, ESR, CRP, RF, lipid profile, fasting and 2-hour postprandial blood sugar.

\section{The procedure:}

(1) Ultrasonographic scanning of posterior tibial nerve bilaterally at popliteal fossa, $5 \mathrm{~cm}$ above tarsal tunnel, proximal inlet of tunnel and within the tunnel.
(2) Electrophysiological studies that included sensory conduction studies (SCS), motor conduction studies (MCS), F-response of posterior tibial nerve and H-reflex.

Cross sectional area of the tibial nerve was the most widely accepted for diagnosis of posterior tibial nerve neuropathy by tracing the posterior tibial nerve inside its hyperechoic rim using the trace function of ultrasound machine.

\section{1- NMUS OF POSTERIOR TIBIAL NERVE AT POPLITEAL FOSSA:}

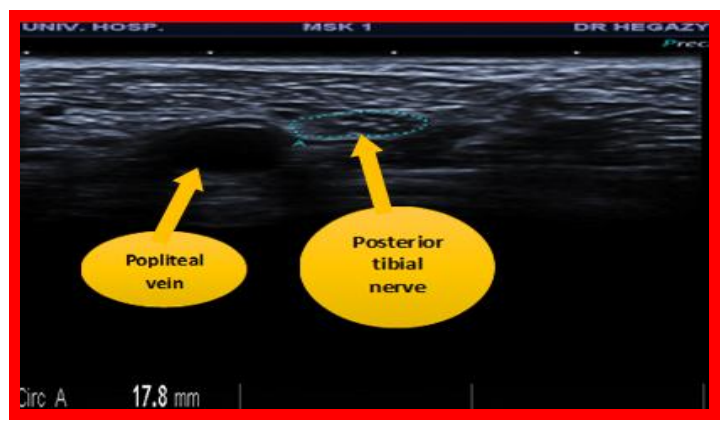

Figure (1): Posterior tibial nerve CSA at popliteal fossa just adjacent to popliteal vein (short axis) and the CSA was $17.8 \mathrm{~mm}$.

\section{2-NMUS OF POSTERIOR TIBIAL NERVE 5 CM ABOVE THE TARSAL TUNNEL:}

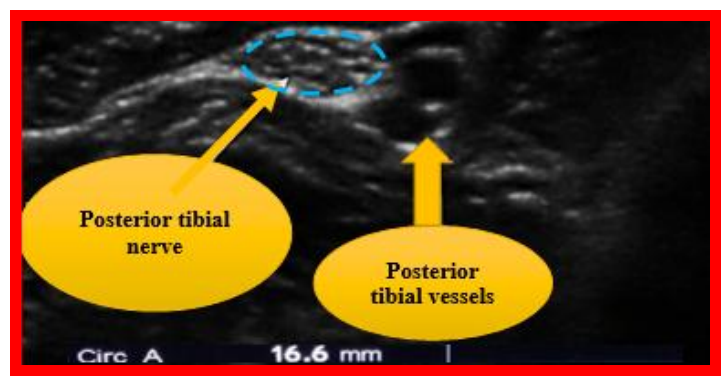

Figure (2): Posterior tibial nerve CSA $5 \mathrm{~cm}$ above the tarsal tunnel (short axis) and the CSA was $16.6 \mathrm{~mm}$.

\section{3-NMUS OF POSTERIOR TIBIAL NERVE AT THE INLET OF THE TARSAL TUNNEL:}

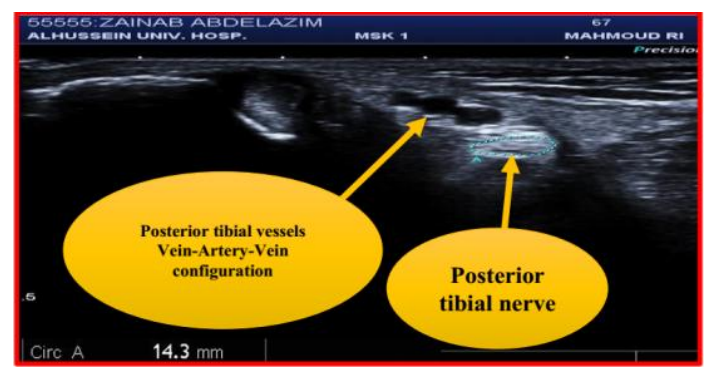

Figure (3): Posterior tibial nerve CSA at the inlet of the tarsal tunnel (short axis) and the CSA was $14.3 \mathrm{~mm}$. 


\section{4-NMUS OF POSTERIOR TIBIAL NERVE WITH IN THE TUNNEL:}

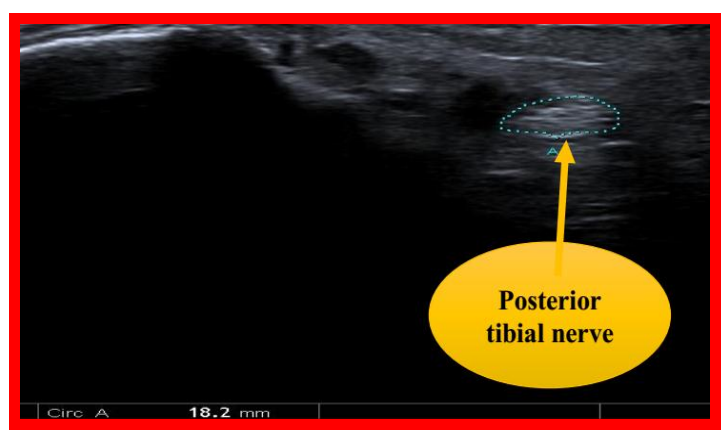

Figure (4): Posterior tibial nerve CSA within the tunnel (short axis) and the CSA was $18.2 \mathrm{~mm}$.

Statistical analysis: Data were analyzed using Statistical Program for Social Science (SPSS) version 15.0. Quantitative data were expressed as mean \pm standard deviation (SD). Qualitative data were expressed as frequency and percentage.

The following tests were done: Independent-samples t-test of significance: was used when comparing between two means. Chi-square test: was used when comparing between nonparametric data. Probability (P-value). P-value $<0.05$ was considered significant. P-value $<0.001$ was considered as highly significant. P-value $>0.05$ was considered insignificant.

\section{RESULTS}

Table (1): Comparison between patients and control groups as regards right posterior tibial nerve motor and sensory conduction studies.

\begin{tabular}{|c|c|c|c|c|c|}
\hline \multicolumn{3}{|l|}{ Variables } & $\begin{array}{c}\text { Patients } \\
\text { group } \\
(\mathbf{N}=\mathbf{3 0})\end{array}$ & $\begin{array}{c}\text { Control } \\
\text { group } \\
(\mathbf{N}=\mathbf{3 0})\end{array}$ & p-value \\
\hline \multirow{6}{*}{ 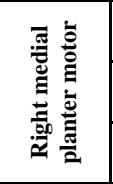 } & \multirow{2}{*}{ Latency } & Mean & 4.9 & 2.4 & \multirow{2}{*}{$<0.001 * *$} \\
\hline & & $\pm \mathrm{SD}$ & \pm 1.4 & \pm 1.06 & \\
\hline & \multirow{2}{*}{ Amplitude } & Mean & 7.7 & 6.7 & \multirow{2}{*}{0.09} \\
\hline & & $\pm \mathrm{SD}$ & \pm 2.9 & \pm 1.9 & \\
\hline & \multirow{2}{*}{$\mathrm{CV}$} & Mean & 46.2 & 48.8 & \multirow{2}{*}{0.08} \\
\hline & & $\pm \mathrm{SD}$ & \pm 7.2 & \pm 3.4 & \\
\hline \multirow{6}{*}{ 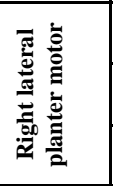 } & \multirow{2}{*}{ Latency } & Mean & 5.1 & 2.7 & \multirow{2}{*}{$<0.001 * *$} \\
\hline & & $\pm \mathrm{SD}$ & \pm 0.9 & \pm 1.4 & \\
\hline & \multirow{2}{*}{ Amplitude } & Mean & 7.7 & 6.7 & \multirow{2}{*}{0.2} \\
\hline & & $\pm \mathrm{SD}$ & \pm 3.7 & \pm 1.5 & \\
\hline & \multirow{2}{*}{$\mathrm{CV}$} & Mean & 47.9 & 48.9 & \multirow{2}{*}{0.07} \\
\hline & & $\pm \mathrm{SD}$ & \pm 4.1 & \pm 3.8 & \\
\hline \multirow{4}{*}{ 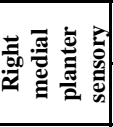 } & \multirow{2}{*}{ Latency } & Mean & 2.8 & 2.6 & \multirow{2}{*}{0.2} \\
\hline & & $\pm \mathrm{SD}$ & \pm 0.6 & \pm 0.5 & \\
\hline & \multirow{2}{*}{ Amp } & Mean & 15.9 & 19.2 & \multirow{2}{*}{$0.002 *$} \\
\hline & & $\pm \mathrm{SD}$ & \pm 3.6 & \pm 4.5 & \\
\hline \multirow{4}{*}{$\begin{array}{c}\text { Right } \\
\text { lateral } \\
\text { planter } \\
\text { sensory }\end{array}$} & \multirow{2}{*}{ Latency } & Mean & 2.6 & 2.7 & \multirow{2}{*}{0.5} \\
\hline & & $\pm \mathrm{SD}$ & \pm 0.6 & \pm 0.4 & \\
\hline & \multirow{2}{*}{ Amp } & Mean & 15.7 & 20.8 & \multirow{2}{*}{$<0.001 *$} \\
\hline & & \pm SD & \pm 3.4 & \pm 3.3 & \\
\hline
\end{tabular}

\section{This table showed:}

Highly statistical significant difference (pvalue $<0.001$ ) between patients and control as regards right medial planter latency.

Highly statistical significant difference (pvalue $<0.001$ ) between patients and control as regards right lateral planter motor latency.

Highly statistical significant difference ( $\mathrm{p}$ value < 0.001) between patients and control as regards right lateral planter sensory amplitude.

Statistically significant difference ( $p$-value $<0.05$ ) between patients and control as regards right medial planter sensory amplitude.

Table (2): Comparison between patients and control groups as regards NMUS of right posterior tibial nerve.

\begin{tabular}{|c|c|c|c|c|}
\hline \multicolumn{2}{|c|}{$\begin{array}{l}\text { Groups } \\
\text { NMUS of Rt. Posterior } \\
\text { tibial n. }\end{array}$} & \multirow{2}{*}{$\begin{array}{c}\begin{array}{c}\text { Patients } \\
\text { group } \\
(\mathbf{N}=\mathbf{3 0})\end{array} \\
21.7 \\
\end{array}$} & \multirow{2}{*}{$\begin{array}{c}\begin{array}{c}\text { Control } \\
\text { group } \\
(\mathbf{N}=\mathbf{3 0})\end{array} \\
24.1 \\
\end{array}$} & \multirow{3}{*}{$\begin{array}{c}\text { p-value } \\
0.001^{*}\end{array}$} \\
\hline \multirow{2}{*}{$\begin{array}{l}\text { Popliteal } \\
\text { Fossa }\end{array}$} & Mean & & & \\
\hline & $\pm \mathrm{SD}$ & \pm 2.5 & \pm 2.9 & \\
\hline \multirow{2}{*}{$\begin{array}{l}\text { CSA } 5 \mathrm{~cm} \\
\text { above tunnel }\end{array}$} & Mean & 16.02 & 15.2 & \multirow{2}{*}{0.2} \\
\hline & $\pm \mathrm{SD}$ & \pm 2.2 & \pm 2.8 & \\
\hline \multirow{2}{*}{ Proximal inlet } & Mean & 16.5 & 11.06 & \multirow{2}{*}{$<0.001^{*}$} \\
\hline & \pm SD & \pm 12.3 & \pm 2.9 & \\
\hline \multirow{2}{*}{$\begin{array}{l}\text { Within the } \\
\text { tunnel }\end{array}$} & Mean & 16.4 & 10.4 & \multirow{2}{*}{$<0.001 *$} \\
\hline & $\pm \mathrm{SD}$ & \pm 3.2 & \pm 2.8 & \\
\hline
\end{tabular}

\section{This table showed:}

Highly statistical significant difference ( $\mathrm{p}$ value < 0.001) between patients and control as regards NMUS of Rt PTN CSA at proximal inlet, within the tunnel.

Statistically significant difference (p-value $<0.05$ ) between patients and control as regards NMUS of Rt PTN CSA at popliteal Fossa.

No statistical significant difference (p-value $>0.05$ ) between patients and control as regards NMUS of Rt PTN CSA $5 \mathrm{~cm}$ above Tunnel.

Table (3): Correlation study between right medial and lateral planter motor latencies, amplitudes with CSA of rt PTN at popliteal fossa, proximal inlet, within the tunnel.

\begin{tabular}{|l|c|c|}
\hline \multicolumn{1}{|c|}{ Right Medial planter motor } & (r) & p-value \\
\hline Popliteal fossa vs latency & 0.07 & 0.7 \\
\hline 5 cm above the tunnel vs latency & 0.08 & 0.8 \\
\hline Proximal inlet vs latency & 0.3 & $<0.05$ \\
\hline Within the tunnel vs latency & 0.05 & $<0.05$ \\
\hline Popliteal fossa vs amplitude & 0.05 & 0.7 \\
\hline 5 cm above the tunnel vs amplitude & 0.2 & 0.08 \\
\hline Proximal inlet vs amplitude & -0.2 & $<0.05$ \\
\hline Within the tunnel vs amplitude & -0.06 & $<0.05$ \\
\hline
\end{tabular}


This table showed:

Statistically significant (p-value $<0.05)$ positive correlation between it PTN CSA at proximal inlet vs it med planter motor latency and it PTN CSA within the tunnel vs it medial planter motor latency.

Statistically significant (p-value $<0.05)$ negative correlation between rt PTN CSA at proximal inlet vs rt med planter motor amplitude and rt PTN CSA within the tunnel vs $\mathrm{rt}$ medial planter motor amplitude.

\section{DISCUSSION}

This study aimed to evaluate neuromuscular ultrasound versus electrophysiological studies in assessment of posterior tibial nerve neuropathy in rheumatoid arthritis patients. Thirty feet of RA patients having pain and burning sensation in their feet and hence suspected to have posterior tibial nerve neuropathy (tarsal tunnel syndrome). They were examined by electrophysiological studies and by neuromuscular ultrasound (NMUS). All patients (100\%) fulfilled the inclusion criteria that all of them were rheumatoid arthritis patients. They were diagnosed according to American College of Rheumatology (ACR) 2010 criteria and ACR criteria for classification of rheumatoid arthritis (1987). They had pain and/or paresthesia in the sole of foot. They were recruited from Physical Medicine, Rheumatology and Rehabilitation Department at Al Hussein and Sayed Galal University Hospitals.

Similar inclusion criteria were adopted by Mondelli et al. ${ }^{(8)}$, who suggested inclusion of cases, based on clinical history and symptoms suggesting any kind of paresthesia and/or pain in all or part of the foot supplied by the plantar nerves, were included. However, Lanzillo et al. ${ }^{(9)}$ considered that pain and paresthesia in rheumatoid arthritis patients could be due to other causes as referred pain of arthritis or tendinitis or even plantar fasciitis and be misinterpreted by patients complains as being related to a neurological problem. This could lead clinicians to over- or underestimate the incidence of clinical symptoms.

Regarding to the mean age, in the current study it was $45.4 \pm 8.3$ years old. Samarawickrama et al. $^{(7)}$ in their study on a retrospective analysis of nerve ultrasound changes in electrophysiologically confirmed that mean age was 50.5 years old. In another study by Cartwright et al. ${ }^{(10)}$ found that the mean age of the studied patient group was 45.9 years old.

In the present study, regarding to sex, there was female predominance $(90 \%)$ and male about $(10 \%)$ that was in agreement with the study done by Smith et al. ${ }^{(11)}$ who studied one hundred and seventy six patients with RA with female predominance.

As regards duration of the disease, in the current study the mean duration of symptoms was $5.9 \pm 5.4$ months. El Shazly et al. ${ }^{(12)}$ stated that the mean duration of symptoms before surgery was $13.4 \pm 1.3$ months.

In the present study, concerning DAS score ranged from 1.6 to 5.3 with a mean of $3.2 \pm 1.08$ in the patient group. In a study by Mi Kyung et al. ${ }^{(13)}$ found that DAS score $3.1 \pm 1.6$ in the RA patients with peripheral neuropathy group, which was near to our results.

Regarding posterior tibial nerve neuropathy symptoms, of group1 (RA patients), there were tingling in 12 feet $(40 \%)$, numbness in 10 feet $(33.3 \%)$, burning sensation in 8 feet $(26.6 \%)$ that slightly agreed with the study done by Ibrahim et al. ${ }^{(14)}$ They studied thirty patients and commented that thirty feet of rheumatoid arthritis patients had pain and burning sensation in their feet and hence suspected to have TTS.

Another objective sign in our study was the triple compression stress test (TCST) being positive in $30(100 \%)$ cases, that slightly agreed with the study done by Abouelela and Zohiery ${ }^{(15)}$ who studied 65 feet and found that $61(93.8 \%)$ had an increase in intensity of symptoms with the clinical TCST.

In the current study, there was no statistical significance between the patients group and the control group concerning the H-reflex and F-wave latencies and sural nerve SCVs. This agreed with the study of Ibrahim et al. ${ }^{(14)}$ who commented that there was no statistically significant difference between the patients and control groups regarding the H-reflex, Fwave latencies and sural nerve SCVs.

In the current study we did not use needle EMG as part of the electrophysiological study for the diagnosis of TTS. This agreed with the review of Patel et al. ${ }^{(16)}$ where none of the 317 articles reviewed mentioned the use of needle EMG in diagnosis of TTS. Another reason for not using needle EMG was a difficulty of localization and interpretation. Intrinsic foot muscles commonly showed increased insertional activity and occasionally fibrillation potentials that had been thought to be due to everyday wear and tear on the foot.

In the present study, nerve conduction findings in RA patients (group1) and control 
subjects (group 2) regarding the posterior tibial nerve (medial and lateral plantar motor branches) parameters. There was highly statistical difference between the two groups as regards motor latency was $5.3 \pm 1.1 \mathrm{~ms}$ in RA patients (group1) and $2.9 \pm$ 1.6 in control subjects (group 2). This agreed with the nerve conduction results of Abd El-Samad et al. ${ }^{(17)}$ who reported a highly significant $(\mathrm{P} \leq 0.001)$ changes in the motor and sensory nerve studies regarding mean values of distal latency, amplitude, and conduction velocity in RA patients compared with healthy individuals.

In the present study, there were highly significance difference as regards medial and lateral planter sensory amplitude between the two groups. Amplitude was $15.7 \pm 3.4$ in RA patients (group1) and $20.8 \pm 3.3$ in control subjects (group 2). This agreed with the review of Lanzillo et al. ${ }^{(9)}$ who studied 40 feet of rheumatoid arthritis patients and found that sensory potential amplitude was significantly decreased at the medial malleolus in $37.5 \%$ of the patients and at the popliteal fossa in $35 \%$. This also agreed with the study done by Ibrahim et al ${ }^{(14)}$ who reported that Sensory affection of the tibial nerve branches was more common than motor affection in tarsal tunnel syndrome.

In our study, posterior tibial nerve ultrasound showed that the mean cross-sectional area at the inlet of the tunnel was $16.7 \pm 2.4 \mathrm{~mm}$ and the mean cross-sectional area in the tunnel was $17.6 \pm 3.3 \mathrm{~mm}$ in group 1 (RA patients). While in group 2 (control subjects), the mean cross-sectional area at the inlet of the tunnel was $11.8 \pm 2.4 \mathrm{~mm}$ and the mean cross-sectional area in the tunnel was $10.1 \pm 2.05 \mathrm{~mm}$. This result was supported by Therimadasamy et al. ${ }^{(18)}$ who found marked enlargement of the tibial nerve at the tunnel itself in a patient with EDX-proven TTS. Also, agreed with the study of Tawfik et al. ${ }^{(19)}$ who reported that the CSA measured within the tunnel was significantly larger in TTS group compared with the controls (mean $13.8 \pm 4.4 \mathrm{~mm} 2$ in controls vs. $20.6 \pm 8.5 \mathrm{~mm} 2$ in patients).

Our results were supported by Fantino ${ }^{(20)}$ who found that US revealed compression elements in $84 \%$ of cases in his study to determine the role of US in posteriomedial tarsal tunnel syndrome (81 cases). Also, agreed with Tawfik et al. (19) who reported that the tunnel CSA and the tunnel-toinlet CSA ratio were the most sensitive, and accurate sonographic parameters to diagnose TTS.
We found highly statistical positive correlation between CSA of the tibial nerve with planter nerves latency and highly statistical negative correlation between CSA of the tibial nerve with planter nerves amplitude. This agreed with review done by Gallarado et $\boldsymbol{a l l}^{(21)}$ who stated that US might add complementary information to neurophysiological studies in the diagnostic work up.

In the other hand, our results did not agree with Abd El-Samad et al. ${ }^{(17)}$ that found that on comparing electrophysiological and US diagnoses of posterior TTS, electrophysiological studies were able to detect more cases of posterior tibial nerve neuropathy. Electrophysiological studies could detect the syndrome in $18(36 \%)$ patients, whereas musculoskeletal ultrasound could detect the syndrome only in $8(16 \%)$ patients. Similar findings were reported by Ibrahim et $\boldsymbol{a l}{ }^{(14)}$ who investigated for posterior TTS in 30 rheumatoid feet by nerve conduction studies, they could detect $28(93.3 \%)$ patients, whereas by musculoskeletal ultrasound only $10(33.3 \%)$ patients could be detected.

\section{CONCLUSION}

Clinical neuropathy occurs in $0.5 \%$ to $85 \%$ of RA patients, and presents in the form of mononeuritis multiplex, sensorimotor neuropathy and entrapment neuropathy.

Compression neuropathies are the most common form of peripheral nervous system involvement in RA and peripheral nerve aff ection was common in the rheumatoid foot, irrespective of the disease activity status.

Clinical examination was the main step in identification of tarsal tunnel syndrome cases especially triple compression stress test due to its great value.

The use of multiple Electrophysiological parameters in diagnosis of tarsal tunnel syndrome was highly appreciated than the use of a single electrodiagnostic test and neurophysiological studies have long been considered to be an extension of the clinical examination.

Sensory affection of the tibial nerve branches was more common than motor affection in tarsal tunnel syndrome.

Neuromuscular ultrasound became an attractive complement to electrodiagnostic (EDX) studies in the evaluation of posterior tibial nerve neuropathy.

Neuromuscular ultrasound could detect pathologies that may predispose or lead to entrapment neuropathy. 
US had a complementary role in the diagnosis of peripheral nerve entrapments. US had the advantage of excellent resolution of superficial nerves. In addition, the dynamic nature of image acquisition made it a natural fit for the neuromuscular and electrodiagnostic clinics.

The combined use of electrophysiology with neuromuscular ultrasonography further confirms the diagnosis of posterior tibial nerve neuropathy.

\section{REFERENCES}

1. Loveday DT, Jackson GE, Geary NP (2012): The rheumatoid foot and ankle: current evidence. Foot and Ankle Surgery, 18: 94- 102.

2. Satish VK, Rakhil SY, Bhagyadhan AP (2017): Vasculitic Neuropathy. In: Electromyography and Neuromuscular Disorders. 2nd edition, pp: 337-350.

3. Dani K, Ramachandran R, Capella A (2005): Neuropathies in the Rheumatoid patient: a case of the heavy hand. Scott Med J., 50: $125-6$.

4. Bonauto DK, Silverstein BA, Fan ZJ, Smith CK, Wilcox DN (2008): Evaluation of a symptom diagram for identifying tarsal tunnel syndrome. Occupational Medicine, 58: 561-566.

5. Ghosh SK, Raheja S, Tuli A (2013): Potential sites of compression of tibial nerve branches in foot: a cadaveric and imaging study. Clin Anat., 26(6): 768-779.

6. Preston D, Shapiro B (2013): Tarsal tunnel syndrome. In: Electromyography and neuromuscular disorders Philadelphia, 3rd Edition, pp: 365 -372.

7. Samarawickrama D, Aravinda K, Therimadasmy, Cheun Chan Y, Vijayan J (2016): Nerve ultrasound in electro physiologically verified tarsal tunnel syndrome. Muscle Nerve, 53: 906-912.

8. Mondelli M, Morana P, Padua L (2004): An electrophysiological severity scale in tarsal tunnel syndrome. Acta Neurol Scand, 109: 284-9.

9. Lanzillo B, Pappone N, Crisci C (1999): Subclinical peripheral nerve involvement in patient with rheumatoid arthritis. Arthritis Rheum., 42: 1304-5.

10. Cartwright MS, Passmore LV, Yoon JS et al. (2008): Cross-sectional area reference values for nerve ultrasonography. Muscle Nerve, 37(5): 566Y571.
11. Smith HS, Smith AR, Seidner P (2011): Painful rheumatoid arthritis. Pain Physician, 14: E427-E458.

12. El Shazly O, El Shazly A, Desouky A, El Zohiery AK, Sakr H (2011): Bases of endoscopic tarsal tunnel release: anatomical and ultrasonographic study with a preliminary clinical report. Surg Radiol Ana.t, 33(10): 929-936.

13. Mi K S, Dae-Yul K, Jisun Y, Dae H P, YongGil K (2014): Assessment of Peripheral Neuropathy in Patients with Rheumatoid Arthritis Who Complain of Neurologic Symptoms. Ann Rehabil Med., 38(2): 249-255.

14. Ibrahim I, Medani S, El-Hameed M, Imam M, Shaaban M (2013): Tarsal tunnel syndrome in patients with rheumatoid arthritis, electrophysiological and ultrasound study. Alexandria J Med., 49: 95 -104.

15. Abouelela A, Zohiery AK (2012): The triple compression stress test for diagnosis of tarsal tunnel syndrome. The Foot, 22 (2012): 146-149

16. Patel T, Gaines $K$, Malamut $R$ et al. (2005): Usefulness of electrodiagnostic techniques in the evaluation of suspected tarsal tunnel syndrome: an evidence-based review. Muscle Nerve, 32: 236-40.

17. Abd El-Samad IE, Samar GS, Alaa AL, Ashraf AZ, Amira TE (2016): Foot neuropathy in rheumatoid arthritis patients: clinical, electrophysiological, and ultrasound studies. Egypt Rheumatol Rehabil., 43: 85-94

18. Therimadasamy AK, Seet RC, Kagda YH, Wilder-Smith EP (2011): Combination of ultrasound and nerve conduction studies in the diagnosis of tarsal tunnel syndrome. Neurology India, 59 (2): 296-297.

19. Tawfik EA, El Zohiery AK, Abouelela A (2016): Proposed Sonographic Criteria for the Diagnosis of Idiopathic Tarsal Tunnel Syndrome. archives of physical medicine and rehabilitation, 1:10-14.

20. Fantino O (2014): Role of ultrasound in posteromedial tarsal tunnel syndrome: 81 cases. J Ultrasound, 17(2): 99-112.

21. Gallardo E, Noto YI, Simon NG (2015): Ultrasound in the diagnosis of peripheral neuropathy: structure meets function in the neuromuscular clinic. J Neurol Neurosurg Psychiatry. 10:1-9. 